\title{
CBIR Feature Vector Dimension Reduction with Eigenvectors of Covariance Matrix using Row, Column and Diagonal Mean Sequences
}

\author{
Dr. H.B.Kekre \\ Senior Professor \\ Computer Engineering Department, \\ SVKM's NMIMS University, \\ Mumbai, India
}

\author{
Sudeep D. Thepade \\ Ph.D.Research Scholar \& Asst. Prof. \\ Computer Engineering Department, \\ SVKM's NMIMS University, \\ Mumbai, India
}

\author{
Akshay Maloo \\ B.Tech (CE) Student \\ Computer Engineering Department, \\ SVKM's NMIMS University, \\ Mumbai, India
}

\begin{abstract}
Because of the rising demand from wide range of applications the need of faster and better image retrieval techniques is growing day by day. Dimension reduction of CBIR feature vectors has gained momentum for swift image retrieval. The paper presents few novel techniques for image retrieval based on principal component analysis (PCA). Here feature vectors are eigenvectors of covariance matrix obtained using the row mean, column mean, forward diagonal mean, backward diagonal mean and mean combinations of database images. Instead of taking all pixels of database images for PCA, proposed CBIR methods use mean vectors, thus dimension of feature vectors used for image retrieval is reduced resulting in faster retrieval. The proposed CBIR techniques are tested on two different image databases, general image database (1000 images spread across 11 categories) and COIL image database (1080 images spread across 15 object categories). For each proposed CBIR technique 55 queries are fired on general image database, 75 queries are fired on COIL image database and net average precision and recall are computed. The experimental results show that proposed CBIR techniques gives the better performance in terms of higher precision and recall values with lesser computational complexity than the conventional PCA based CBIR using complete image data.
\end{abstract}

\section{Keywords}

CBIR; PCA; Eigenvectors; Row Mean; Column Mean; Diagonal Mean

\section{INTRODUCTION}

The large numbers of images are being generated from a variety of sources (digital camera, digital video, scanner, the internet etc.) which have posed technical challenges to computer systems to store/transmit and index/manage image data effectively to make such collections easily accessible. Image compression deals with the challenge of storage and transmission, where significant advancements have been made $[1,4,5]$. The challenge to image indexing is studied in the context of image databases [2,6,7,10,11], which has become one of the promising and important research area for researchers from a wide range of application disciplines like art galleries [12,14], museums, archaeology [3], architecture design [8,13], geographic information systems [5], weather forecast [5,22], medical imaging [5,18], trademark databases $[21,23]$, criminal investigations [24,25], image search on the Internet $[9,19,20]$.

\subsection{Content Based Image Retrieval}

Kato et. al. [4] used the term content based image retrieval (CBIR) for the first time in literature, to describe their experiments into automatic retrieval of images from a database by color and shape feature. The typical CBIR system performs two major tasks $[16,17]$. The first one is feature extraction (FE), where a set of features, called feature vector, is generated to accurately represent the content of each image in the database [31,32]. The second task is similarity measurement ( $\mathrm{SM})$, where a distance between the query image and each image in the database using their feature vectors is used to retrieve the top "closest" images [16,17,26,30]. Many approaches have been proposed for feature extraction in CBIR. The feature extraction in spatial domain includes the CBIR techniques based on histograms [5], BTC [1,2,16], VQ [21,25,26]. The transform domain feature extraction methods are widely used in CBIR [17,23,24]. In all these spatial and transform domain CBIR techniques the feature vector space for all images in database plays major role in deciding retrieval efficiency.

Efforts have also been made to increase the speed of content-based image retrieval, the system works slower mainly because of the high dimensionality of the feature space, typically hundreds to thousands [29]. Raymond Ng and Sedighian [34] made direct use of eigenimages, a method from face recognition [35], to carry out the dimension reduction. Faloutsos and Lin [36] and Chandrasekarenet al. [37] used principal component analysis (PCA) to perform the dimension reduction in feature spaces. Experimental results in these works show that most real image feature sets can be considerably reduced in dimension without significant degradation in retrieval performance.

Many current CBIR systems use Euclidean distance [1-3,8-14] on the extracted feature set as a similarity measure. The Direct Euclidian Distance between image P and query image Q can be given as equation 1, where Vpi and Vqibe the feature vectors of image $P$ and Query image Q respectively with size ' $n$ '.

$$
E D=\sqrt{\sum_{i=1}^{n}(V p i-V q i)^{2}}
$$

\section{PRINCIPAL COMPONENT ANALYSIS (PCA) $[27,28,40]$}

CBIR is discriminating the images from image database into several categories. The database images belonging to same category may differ in lighting conditions, noise etc., but are not 
completely random and inspite of their differences there may present some patterns. Such patterns could be referred as principal components. Principal Component Analysis (PCA) is a mathematical tool used to extract principal components of original image data. These principal components may also be referred as Eigenimages.

PCA is generally used for face recognition. The idea of using principal components to represent human faces was developed by Sirovich and Kirby [38] in 1987 and used by Turk and Pentland [27] in 1991 for face detection and recognition. The Eigenface approach is considered by many to be the first working facial recognition technology. PCA can be used to transform each original image from database into its corresponding eigenimage. An important feature of PCA is that any original image from the image database can be reconstructed by combining the eigenimages. Even only some part of eigenimage can be used to reconstruct an approximate of the original image. PCA can do the jobs like prediction, redundancy removal, feature extraction, data compression, etc. So using PCA for image retrieval becomes obvious.

\section{PCA ALGORITHM $[35,40]$}

The various steps to calculate eigenimages are:

\section{A. Prepare the data}

A 2-D database image can be represented as 1-D vector by concatenating each row (or column) into a long thin vector. Let's suppose we have $\mathrm{M}$ vectors of size $\mathrm{N}$ (= rows of image $\times$ columns of image) representing a set of sampled images.

Then the training set becomes: $\Gamma_{1}, \Gamma_{2}, \Gamma_{3} \ldots . . \Gamma_{\mathrm{M}}$.

\section{B. Subtract the mean}

The average matrix $\Psi$ has to be calculated, then subtracted from the original images $(\Gamma i)$ and the result stored in the variable $\Phi \mathrm{i}$ :

$$
\begin{aligned}
& \Psi=\frac{1}{M} \sum_{n=1}^{M} \Gamma_{n} \\
& \Phi_{i}=\Gamma_{i}-\Psi
\end{aligned}
$$

C. Calculate the co-variance matrix

In this step the covariance matrix A is calculated according to:

$$
\mathrm{A}=\Phi^{\mathrm{T}} \Phi
$$

D. Calculate the eigenvectors and eigenvalues of the covariance matrix: In this step, the eigenvectors (eigenvectors) $\mathrm{Xi}$ and the corresponding eigen values $\lambda \mathrm{i}$ should be calculated.

\section{E. Calculate eigenimages}

$$
[\Phi] X_{i}=f_{i}
$$

where $X_{i}$ are eigenvectors and $f_{i}$ are eigenimages.

\section{F. Classifying the faces}

The new image is transformed into its eigenimage components. The resulting weights form the weight vector $\Omega_{\text {new }}$ :

$$
\Omega_{\text {new }}=\left[\begin{array}{llll}
\omega_{1} \omega_{2} \omega_{3} & \ldots & \omega_{M^{\prime}}
\end{array}\right]
$$

where $\omega_{k}$ indicates the addition of element wise product of image $\Gamma_{\text {new }}$ with eigenimage $f_{k}$.

$$
\omega_{k}=<\Gamma_{\text {new }} \mid f_{k}>, \quad \mathrm{k}=1,2 \ldots . \mathrm{M}
$$

The Euclidean distance [11-14] between two weight vectors $\mathrm{d}\left(\Omega_{\mathrm{i}}, \Omega_{\mathrm{j}}\right)$ provides a measure of similarity between the corresponding images $\mathrm{i}$ and $\mathrm{j}$.

\section{ROW MEAN (RM)/COLUMN MEAN (CM) $[10,17,41]$}

The row mean vector is the set of averages of the intensity values of the respective rows. The column mean vector is the set of averages of the intensity values of the respective columns. If fig. 1 is representing the sample image with $\mathrm{n}$ rows and $\mathrm{n}$ columns, the row and column mean vectors for this image will be as given below.

Row Mean Vector $=$

[Avg(Row 1), $\operatorname{Avg}($ Row 2), ..., $\operatorname{Avg}($ Row n)]

Column Mean Vector $=$

$$
\text { [Avg(Col. 1), } \operatorname{Avg}(\text { Col. 2), ...., Avg(Col. n)] }
$$

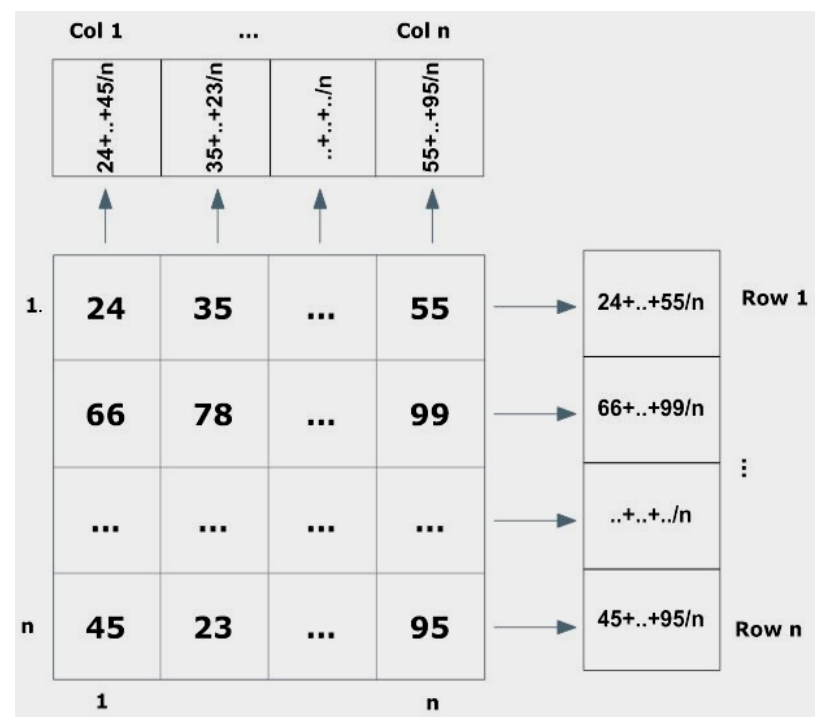

Fig. 1 Row Mean and Column Mean of Sample Image

\section{FORWARD /BACKWARD DIAGONAL MEAN [41]}

The forward diagonal mean (FDM) vector is the set of averages of the intensity values of the diagonal elements in the direction of a forward slash. The backward diagonal mean (BDM) vector is the set of averages of the intensity values of the diagonal elements in the direction of a backward slash. If fig. 2 is representing the sample image with 4 rows and 4 columns, the FDM vector is shown and fig. 3 BDM vector is shown, and the final vector for this image will be as given below.

Forward Diagonal Mean Vector $=$

$$
\text { [Avg(FDM 1), Avg(FDM 2), ..., Avg(FDM n-1)] }
$$

Backward Diagonal MeanVector $=$

$$
\text { [Avg(BDM. 1), Avg(BDM. 2), ..., Avg(BDM. n-1)] }
$$




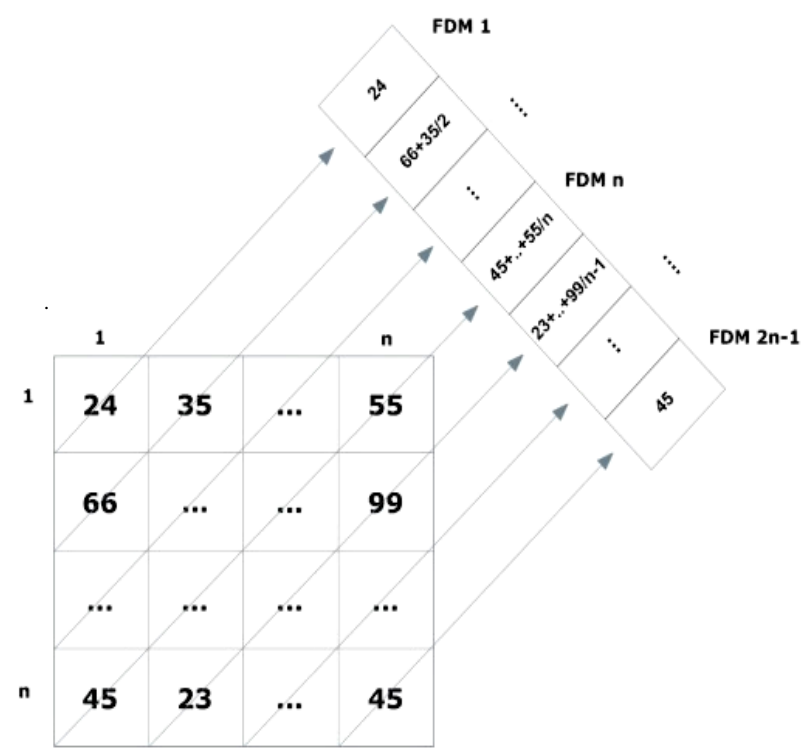

Fig.2 Forward Diagonal Mean of Sample Image

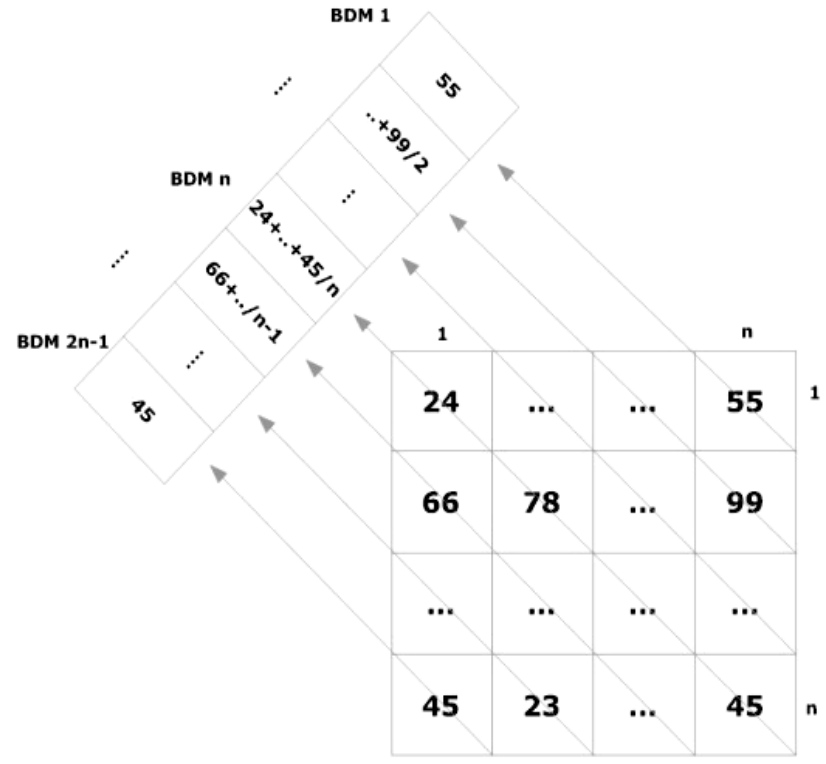

Fig.3 Backward Diagonal Mean of Sample Image

\section{PROPOSED CBIR TECHNIQUES}

In proposed CBIR techniques eigenimages are found for all images in the database using PCA. The eigen sequences are generated using various image data forms as listed below.

- All image pixels,

- Row Mean of image,

- Column mean of image,

- Combination row and column mean,

- Forward diagonal mean of image,

- Backward diagonal mean of image,

- Combination of diagonal means.

All these PCA eigenimage features are generated by considering gray and color (RGB) images from the database resulting into total 14 different CBIR techniques. The advantage of using the means of images over the complete pixel data of image is reduced complexity of image retrieval technique.

\section{COMPLEXITY ANALYSIS}

The computational complexity of applying PCA on complete images is drastically reduced in proposed techniques. The analysis of abridged complexity in feature extraction as well as query execution for the discussed CBIR methods in terms of number of additions and multiplications is shown in table 1 . The percentage reduction in complexity of PCA applied to row mean, column mean and diagonal mean based CBIR techniques as compared to PCA applied on complete image data is shown in table 2.

Table 1. Computational Complexity of proposed CBIR techniques using PCA

\begin{tabular}{|c|c|c|c|c|}
\hline \multirow{2}{*}{$\begin{array}{c}\text { PCA applied } \\
\text { on }\end{array}$} & \multicolumn{2}{|c|}{ Feature Extraction } & \multicolumn{2}{c|}{ Query Execution } \\
\cline { 2 - 5 } & Additions & Multiplications & Additions & Multiplications \\
\hline All & $285,196,288$ & $285,212,672$ & $268,484,608$ & $268,484,606$ \\
\hline Row Mean & 17,280 & 17,408 & 16,768 & 16,766 \\
\hline Column Mean & 17,280 & 17,408 & 16,768 & 16,766 \\
\hline $\begin{array}{c}\text { Row \& Column } \\
\text { mean }\end{array}$ & 69,376 & 69,632 & 66,304 & 66,302 \\
\hline $\begin{array}{c}\text { Forward } \\
\text { Diagonal Mean }\end{array}$ & 68,834 & 69,089 & 65,790 & 65,788 \\
\hline $\begin{array}{c}\text { Backward } \\
\text { Diagonal Mean }\end{array}$ & 68,834 & 69,089 & 65,790 & 65,788 \\
\hline $\begin{array}{c}\text { Forward \& } \\
\text { Backward } \\
\text { Diagonal Mean }\end{array}$ & 275,846 & 276,356 & 261,630 & 261,628 \\
\hline
\end{tabular}

Table 2. Percentage reduction in computational Complexity of proposed CBIR techniques

\begin{tabular}{|c|c|c|c|c|}
\hline \multirow{2}{*}{$\begin{array}{c}\text { PCA applied } \\
\text { on }\end{array}$} & \multicolumn{2}{|c|}{ Feature Extraction } & \multicolumn{2}{c|}{ Query Execution } \\
\cline { 2 - 5 } & Additions & Multiplications & Additions & Multiplications \\
\hline All & 0 & 0 & 0 & 0 \\
\hline Row Mean & 99.993941 & 99.993896 & 99.993755 & 99.993755 \\
\hline Column Mean & 99.993941 & 99.993896 & 99.993755 & 99.993755 \\
\hline $\begin{array}{c}\text { Row\& Column } \\
\text { mean }\end{array}$ & 99.975674 & 99.975586 & 99.975304 & 99.975305 \\
\hline $\begin{array}{c}\text { Forward } \\
\text { Diagonal Mean }\end{array}$ & 99.975864 & 99.975776 & 99.975496 & 99.975497 \\
\hline $\begin{array}{c}\text { Backward } \\
\text { Diagonal Mean }\end{array}$ & 99.975864 & 99.975776 & 99.975496 & 99.975497 \\
\hline $\begin{array}{c}\text { Forward \& } \\
\text { Backward } \\
\text { Diagonal Mean }\end{array}$ & 99.903279 & 99.903105 & 99.902553 & 99.902554 \\
\hline
\end{tabular}

\section{IMPLEMENTATION}

The implementation of the proposed CBIR techniques is done in MATLAB 7.0 using a computer with Intel Core 2 Duo Processor T8100 $(2.1 \mathrm{GHz})$ and 2 GB RAM. The CBIR techniques are tested on the generic image database (created using 839 images from Wang image database [15] with 161 additional images) and COIL image database [33]. The generic image database consists of 1000 variable size images spread across 11 categories of human being, animals, natural scenery and manmade things. The categories and distribution of the images is shown in table 3. 
Table 3.Generic Image Database: Category-wise Distribution

\begin{tabular}{|c|c|c|c|}
\hline Category & Tribes & Buses & Beaches \\
\hline No.of Images & 85 & 99 & 99 \\
\hline Category & Horses & Mountains & Airplanes \\
\hline No.of Images & 99 & 61 & 100 \\
\hline Category & Dinosaurs & Elephants & Roses \\
\cline { 1 - 3 } No.of Images & 99 & 99 & 99 \\
\cline { 1 - 3 } Category & Monuments & Sunrise & \multirow{2}{|}{} \\
\cline { 1 - 3 } No.of Images & 99 & 61 & \multicolumn{2}{|c}{} \\
\cline { 1 - 3 } & &
\end{tabular}

Figure 4 gives the sample generic database images from all categories of images including scenery, flowers, buses, animals, aeroplanes, monuments, tribal people. COIL image database consists of total 1080 images of size $128 \times 128 \times 3$. There are 15 different categories consisting of 72 images in each categories To test the proposed method, from every class five query images are selected randomly. So in all 75 query images are used. Figure 5 gives sample 15 object images of COIL image database.

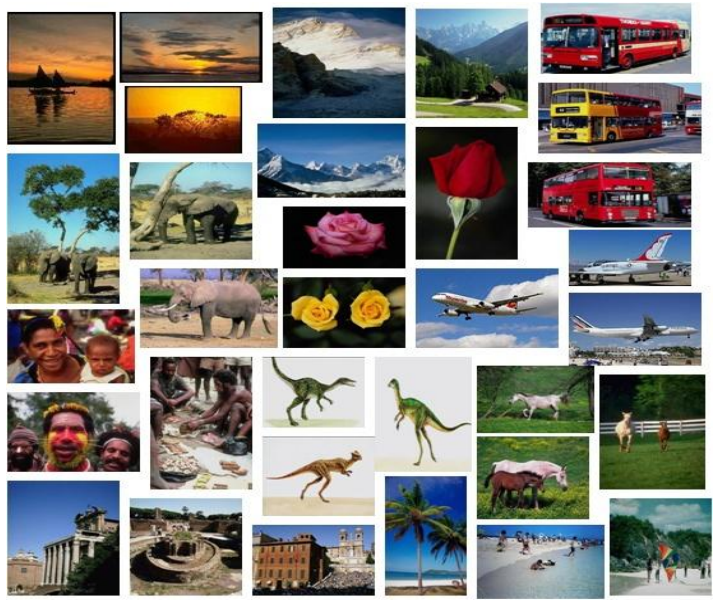

Fig.4.Sample images of Generic Image Database [Image database contains total 1000 images with 11 categories]

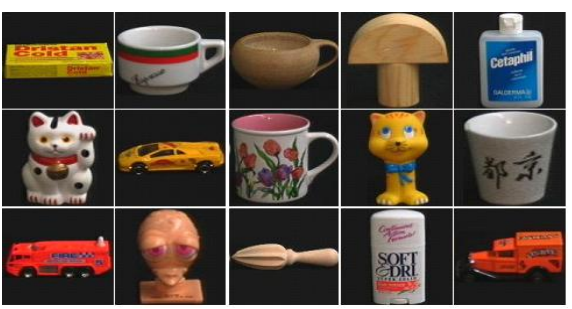

Fig.5. Sample of 15 images of COIL image database the database has 15 categories, for a total of 1,080 images.

To assess the retrieval effectiveness, we have used the precision and recall as statistical comparison parameters [1,2] for the proposed CBIR techniques. The standard definitions of these two measures are given by following equations.

$$
\begin{aligned}
& \operatorname{Pr} \text { ecision }=\frac{\text { Number_of_relevant_images_retrieved }}{\text { Total_number_of_images_retrieved }} \\
& \operatorname{Re} \text { call }=\frac{\text { Number_of_relevant_images_retrieved }}{\text { Total_number_of_relevent_images_in_database }}
\end{aligned}
$$

\section{RESULTS AND DISCUSSION}

For testing the performance of each proposed CBIR technique, per technique 55 queries (5 from each category) are fired on the generic image database of 1000 variable size images spread across 11 categories. And 75 queries (5 from each category) are fired on the COIL image database of 1080 images spread across 15 object classes. The query and database image matching is done using Euclidian distance. The average precision and average recall are computed by grouping the number of retrieved images sorted according to ascending Euclidian distances with the query image.

Figure 6 shows average precision and average recall values plotted against number of retrieved result images for gray COIL image database. Here CBIR using PCA applied on row mean image outperforms the other discussed CBIR techniques with highest precision and recall values at much reduced complexity than PCA applied on complete image data.

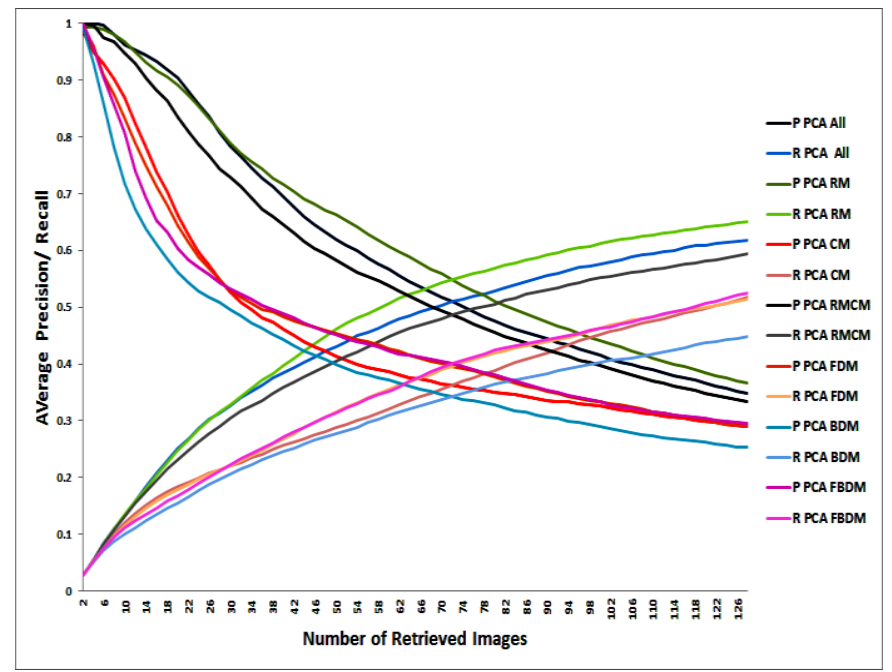

Fig.6. Average Precision/Recall for proposed techniques tested on Gray COIL image database

Figure 7 gives average precision and average recall values plotted against number of retrieved result images for color COIL image database. Here CBIR using PCA applied on combination of row mean image and column mean image gives best performance with highest precision and recall values proving the worth of proposed CBIR techniques. 


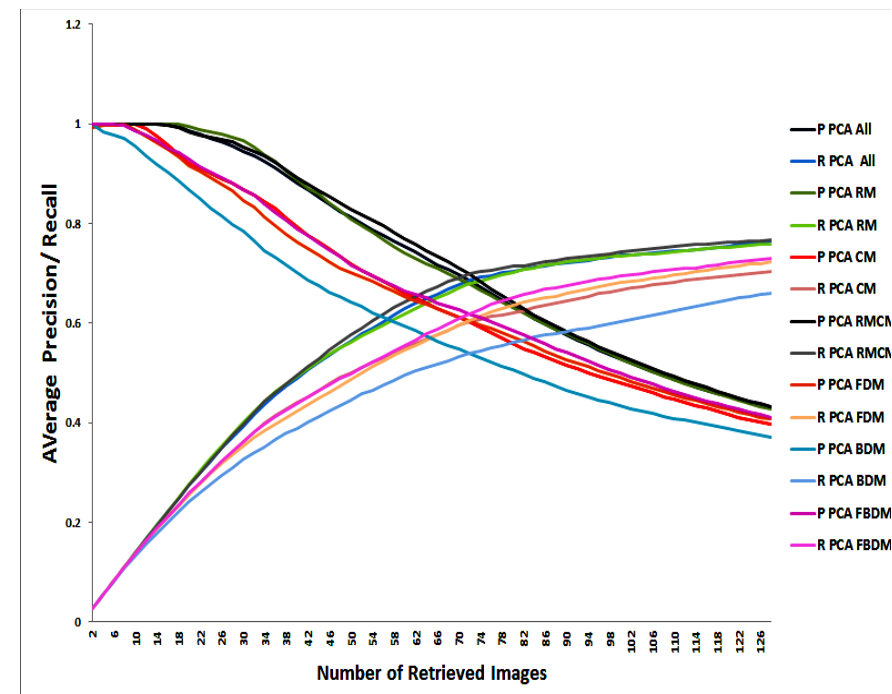

Fig.7. Average Precision/Recall for proposed techniques tested on Color (RGB) COIL image database

The crossover point of precision and recall plays important role in performance comparison of CBIR techniques. More the height of crossover point better the performance of image retrieval method is. Figure 8 shows the comparison of precision-recall crossover points of proposed techniques tested on color COIL image database. Here the techniques based on combination of row mean and column mean and based on row mean image gives almost similar performance as compared to PCA apllied on complete image data but at drastically reduced rate of complexity. So the same performance of image retrieval results can be obtained with faster techniques.

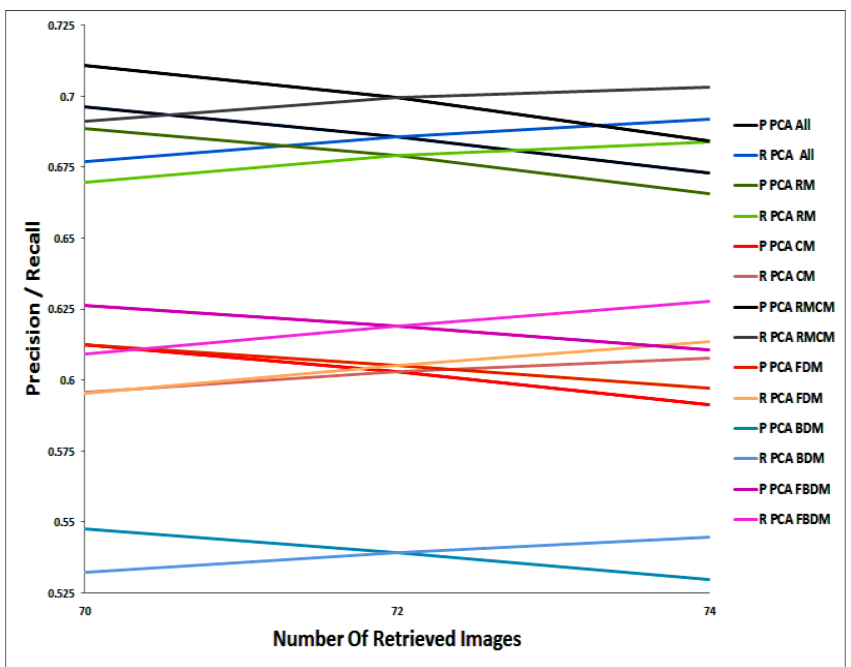

Fig.8. Crossover points of average Precision/Recall for proposed techniques tested on Color COIL image database

Figure 9 shows average precision and average recall values plotted against number of retrieved result images for gray Generic image database. Here best performance is shown by CBIR using PCA on all image data. Further it can be noted that the CBIR using PCA applied on forward diagonal mean is showing nearly same performance as that of PCA applied on complete image data.

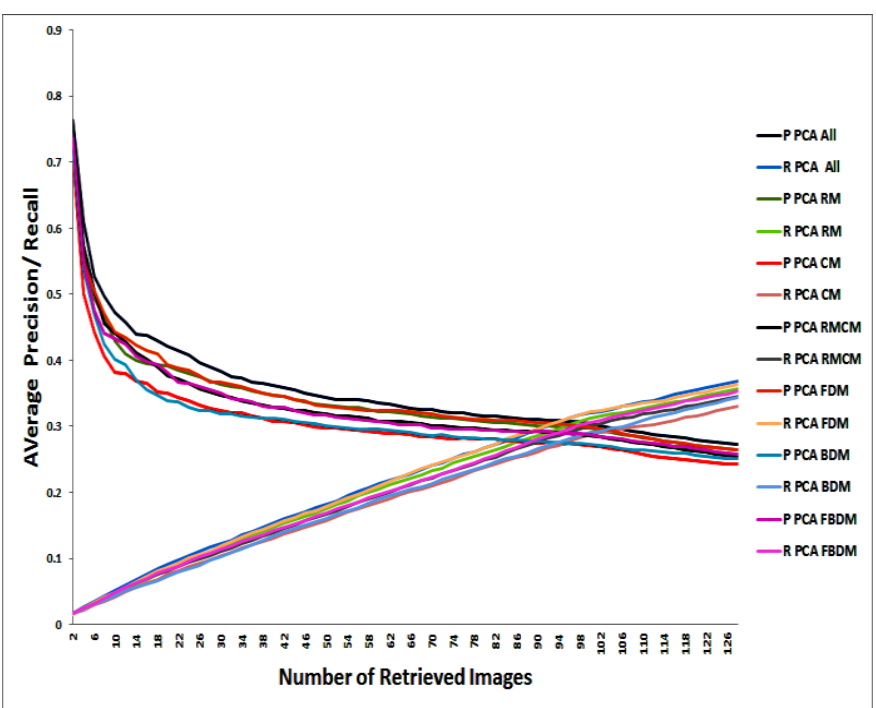

Fig.9. Average Precision/Recall for proposed techniques tested on Gray Generic image database

Average precision and average recall values plotted against number of retrieved result images for gray Generic image database is given in figure 10. Here best performance is shown by CBIR using PCA on all image data. Further it can be noted that the CBIR using PCA applied on row mean image and combination of row mean-column mean image are giving almost similar performance at much reduced complexity.

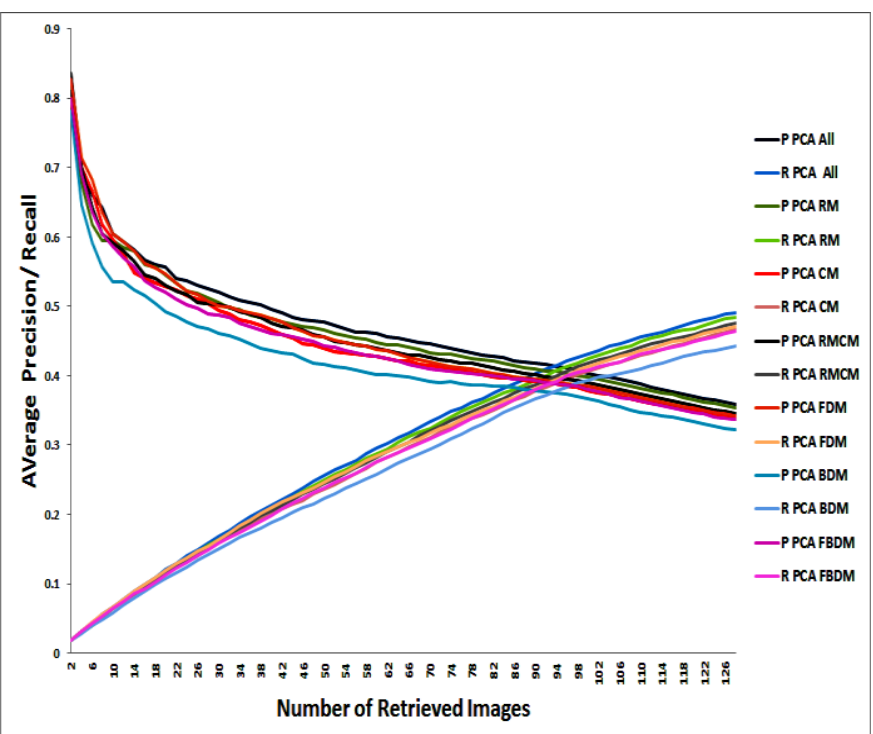

Fig.10. Average Precision/Recall for proposed techniques tested on Color Generic image database

Figure 11 shows the comparison of precision-recall crossover points of proposed techniques tested on color Generic image database. Here it is clearly represented that also CBIR based on PCA with all pixel data gives marginally better performance as compared to other proposed CBIR techniques. 


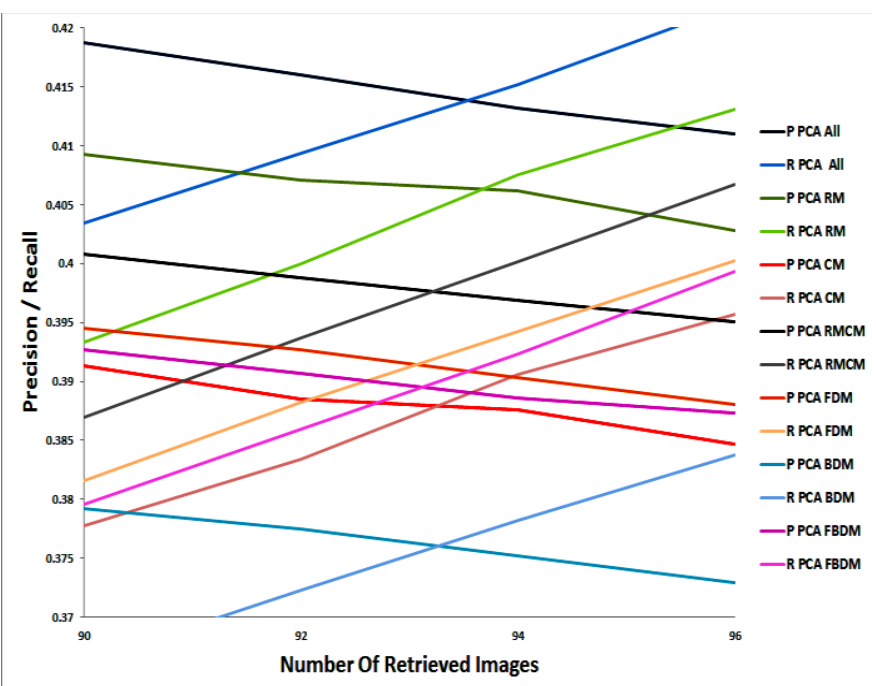

Fig.11. Crossover points of average Precision/Recall for proposed techniques tested on Color Generic image database

Figure 12 is indicating the comparison of crossover point heights for all proposed CBIR techniques tested on COIL image database. The graph clearly reflects that for every CBIR technique discussed here the performance is improved in considering PCA applied on color data than applying PCA on gray data. In gray COIL image database the CBIR techniques with combination of row mean image gives the best performance as indicated by highest crossover point value with less complexity than considering all pixel data. In color COIL image database the image retrieval technique with combination of row mean and column mean image data gives best performance at drastically reduced complexity. This proves that the proposed CBIR techniques gives better performance with faster image retrieval.

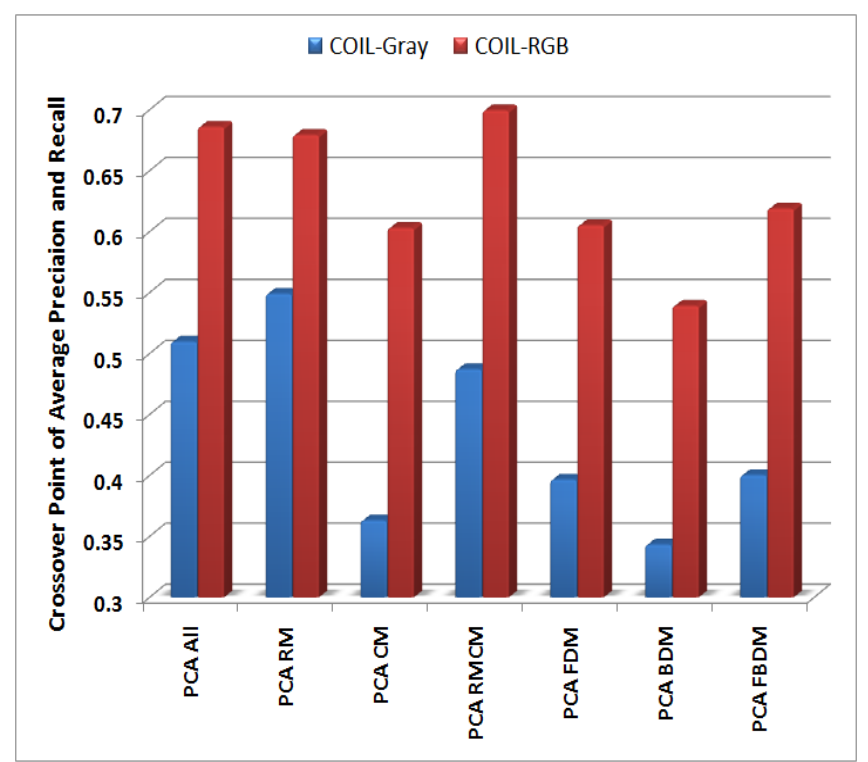

Fig.12. Comparison of Crossover points of average precision and recall for COIL image database

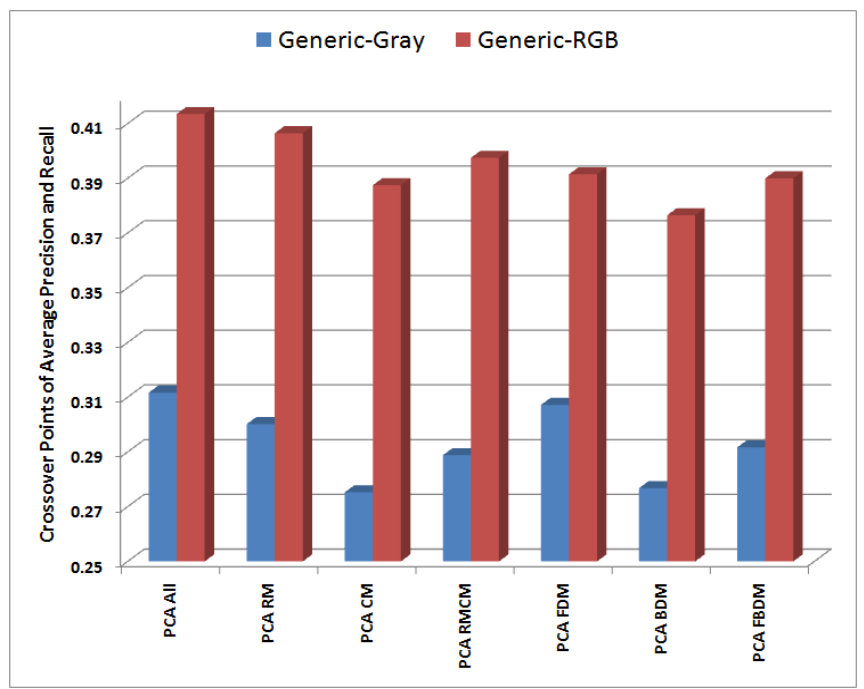

Fig.13. Comparison of Crossover points of average precision and recall for General image database

Figure 13 shows the comparison of proposed CBIR methods on the basis of height of crossover point of average precision and average recall curves. Here also it can be noted easily that the PCA with color data outperforms PCA on gray data for image retrieval. Further the PCA applied on all pixel data gives best performance. Also the performance of other image retrieval techniques based on row mean and combination of row mean-column mean are marginally less than the best. The minor decrease in performance in the row mean and combination of row mean-column mean based image retrieval techniques can be tolerated knowing the tremendous amount of complexity reduced as compared to using PCA on complete pixel data for CBIR.

\section{CONCLUSION}

Today because of the need to search appropriate mages from huge image pools, more précised retrieval techniques are needed. Computational complexity and retrieval efficiency are the key objectives in the image retrieval system. Nevertheless it is very difficult to reduce the computations and improve the performance of image retrieval technique.

The paper has proposed the faster image retrieval techniques based on feature vector dimension reduction with Eigenvectors of Covariance Matrix using Row, Column and Diagonal Mean Sequences. Here the complexity of mage retrieval is tremendously reduced (by $99.9 \%$ ) in proposed techniques as compared to PCA applied on complete image data at the cost of marginal decrease in performance.

\section{REFERENCES}

[1] H.B.Kekre, Sudeep D. Thepade, "Boosting Block TruncationCodingusingKekre's LUV ColorSpace for Image Retrieval", WASET International Journal of Electrical, Computer and System Engineering (IJECSE), Volume 2, Number 3, pp. 172-180, Summer 2008. Available online athttp://www.waset.org/ijecse/v2/v2-3-23.pdf

[2] H.B.Kekre, Sudeep D. Thepade, "Image RetrievalusingAugmented Block TruncationCoding Techniques", ACM International Conference on Advances in 
Computing, Communication and Control (ICAC3-2009), pp. 384-390, 23-24 Jan 2009, Fr. ConceicaoRodrigousCollege of Engg., Mumbai. Is uploaded on online ACM portal.

[3] H.B.Kekre, Sudeep D. Thepade, "Scaling Invariant Fusion of Image Pieces in Panorama Making and Novel Image Blending Technique", International Journal on Imaging (IJI), www.ceser.res.in/iji.html, Volume 1, No. A08, pp. 3146, Autumn 2008.

[4] Hirata K. and Kato T. "Query by visual example - contentbased image retrieval", In Proc. of Third International Conference on Extending Database Technology, EDBT'92, 1992, pp 56-71

[5] H.B.Kekre, Sudeep D. Thepade, "Rendering Futuristic Image Retrieval System", National Conference on Enhancements in Computer, Communication and Information Technology, EC2IT-2009, 20-21 Mar 2009, K.J.Somaiya College of Engineering, Vidyavihar, Mumbai77.

[6] Minh N. Do, MartinVetterli, "Wavelet-Based Texture Retrieval Using Generalized Gaussian Density and KullbackLeibler Distance”, IEEE Transactions On Image Processing, Volume 11, Number 2, pp.146-158, February 2002.

[7] B.G.Prasad, K.K. Biswas, and S. K. Gupta, "Region -based image retrieval using integrated color, shape, and location index", International Journal on Computer Vision and Image Understanding Special Issue: Colour for Image Indexing and Retrieval, Volume 94, Issues 1-3, April-June 2004, pp.193233.

[8] H.B.Kekre, Sudeep D. Thepade, "Creating the Color Panoramic View using Medley of Grayscale and Color Partial Images ", WASET International Journal of Electrical, Computer and System Engineering (IJECSE), Volume 2, No. 3, Summer 2008. Available online at www.waset.org/ijecse/v2/v2-3-26.pdf.

[9] StianEdvardsen, "Classification of Images using color, CBIR Distance Measures and Genetic Programming", Ph.D. Thesis, Master of science in Informatics, Norwegian university of science and Technology, Department of computer and Information science, June 2006.

[10] H.B.Kekre, TanujaSarode, Sudeep D. Thepade, "DCT Applied to Row Mean and Column Vectors in Fingerprint Identification", In Proceedings of International Conference on Computer Networks and Security (ICCNS), 27-28 Sept. 2008, VIT, Pune.

[11] Zhibin Pan, Kotani K., Ohmi T., "Enhanced fast encoding method for vector quantization by finding an optimallyordered Walsh transform kernel", ICIP 2005, IEEE International Conference, Volume 1, pp I - 573-6, Sept. 2005.

[12] H.B.kekre, Sudeep D. Thepade, "Improving 'Color to Gray and Back' using Kekre's LUV Color Space”, IEEE International Advanced Computing Conference 2009 (IACC'09), Thapar University, Patiala, INDIA, 6-7 March 2009. Is uploaded and available online at IEEE Xplore.

[13] H.B.Kekre, Sudeep D. Thepade, "Image Blending in Vista Creation using Kekre's LUV Color Space", SPIT-IEEE Colloquium and International Conference, Sardar Patel Institute of Technology, Andheri, Mumbai, 04-05 Feb 2008.

[14] H.B.Kekre, Sudeep D. Thepade, "Color Traits Transfer to Grayscale Images", In Proc.of IEEE First International

[25] March 2010, The paper will be uploaded on online Springerlink.
Conference on Emerging Trends in Engg. \&Technology, (ICETET-08), G.H.Raisoni COE, Nagpur, INDIA. Uploaded on online IEEE Xplore.

[15] http://wang.ist.psu.edu/docs/related/Image.orig (Last referred on 23 Sept 2008)

[16] H.B.Kekre, Sudeep D. Thepade, "Using YUV Color Space to Hoist the Performance of Block Truncation Coding for Image Retrieval", IEEE International Advanced Computing Conference 2009 (IACC'09), Thapar University, Patiala, INDIA, 6-7 March 2009.

[17] H.B.Kekre, Sudeep D. Thepade, ArchanaAthawale, Anant Shah, PrathmeshVerlekar, SurajShirke,"Energy Compaction and Image Splitting for Image Retrieval using Kekre Transform over Row and Column Feature Vectors", International Journal of Computer Science and Network Security (IJCSNS), Volume:10, Number 1, January 2010, (ISSN: 1738-7906) Available at www.IJCSNS.org.

[18] H.B.Kekre, Sudeep D. Thepade, ArchanaAthawale, Anant Shah, PrathmeshVerlekar, SurajShirke, "Walsh Transform over Row Mean and Column Mean using Image Fragmentation and Energy Compaction for Image Retrieval", International Journal on Computer Science and Engineering (IJCSE), Volume 2S, Issue1, January 2010, (ISSN: 09753397). Available online at www.enggjournals.com/ijcse.

[19] H.B.Kekre, Sudeep D. Thepade, "Image Retrieval using Color-Texture Features Extracted from Walshlet Pyramid", ICGST International Journal on Graphics, Vision and Image Processing (GVIP), Volume 10, Issue I, Feb.2010, pp.9-18, Available online www.icgst.com/gvip/Volume10/Issue1/P1150938876.html

[20] H.B.Kekre, Sudeep D. Thepade, "Color Based Image Retrieval using Amendment Block Truncation Coding with YCbCr Color Space", International Journal on Imaging (IJI), Volume 2, Number A09, Autumn 2009, pp. 2-14. Available online at www.ceser.res.in/iji.html (ISSN: 0974-0627).

[21] H.B.Kekre, TanujaSarode, Sudeep D. Thepade, "ColorTexture Feature based Image Retrieval using DCT applied on Kekre's Median Codebook", International Journal on Imaging (IJI), Volume 2, Number A09, Autumn 2009,pp. 55-65. Available online at www.ceser.res.in/iji.html (ISSN: 0974-0627).

[22] H.B.Kekre, Sudeep D. Thepade, "Image Retrieval using Non-Involutional Orthogonal Kekre's Transform”, International Journal of Multidisciplinary Research and Advances in Engineering (IJMRAE), Ascent Publication House, 2009, Volume 1, No.I, pp 189-203, 2009. Abstract available online at www.ascent-journals.com (ISSN: 09757074)

[23] H.B.Kekre, Sudeep D. Thepade, "Improving the Performance of Image Retrieval using Partial Coefficients of Transformed Image", International Journal of Information Retrieval, Serials Publications, Volume 2, Issue 1, 2009, pp. 72-79 (ISSN: 0974-6285)

[24] H.B.Kekre, Sudeep D. Thepade, ArchanaAthawale, Anant Shah, PrathmeshVerlekar, SurajShirke, "Performance Evaluation of Image Retrieval using Energy Compaction and Image Tiling over DCT Row Mean and DCT Column Mean", Springer-International Conference on Contours of Computing Technology (Thinkquest-2010), BabasahebGawde Institute of Technology, Mumbai, 13-14

[26] H.B.Kekre, Tanuja K. Sarode, Sudeep D. Thepade, VaishaliSuryavanshi,"Improved Texture Feature Based 
Image Retrieval using Kekre's Fast Codebook Generation Algorithm", Springer-International Conference on Contours of Computing Technology (Thinkquest-2010), BabasahebGawde Institute of Technology, Mumbai, 13-14 March 2010, The paper will be uploaded on online Springerlink.

[27] H.B.Kekre, Tanuja K. Sarode, Sudeep D. Thepade, "Image Retrieval by Kekre's Transform Applied on Each Row of Walsh Transformed VQ Codebook", (Invited), ACMInternational Conference and Workshop on Emerging Trends in Technology (ICWET 2010), Thakur College of Engg. And Tech., Mumbai, 26-27 Feb 2010, The paper is invited at ICWET 2010. Also will be uploaded on online ACM Portal.

[28] M. Turk, A. Pentland, " Eigenfaces for Recognition", Journal of Cognitive Neuroscienc , Vol. 3, Number 1, 1991, pp.71-86.

[29] S. Chandrasekaran et al., "An eigenspace update algorithm for image analysis," CVGIP: Graph. Models Image Process. J., 1997.

[30] C. Faloutsos and K. Lin, "Fastmap: A fast algorithm for indexing, data-mining and visualization of traditional and multimedia," in Proc.SIGMOD, 1995, pp. 163-174.

[31] B. Roberto and M. Ornella, "Image retrieval by examples," IEEE Trans.Multimedia, vol. 2, Sept. 2000.

[32] K. Fukunaga, "Introduction to Statistical Pattern Recognition", 2nd ed. New York: Academic, 1990.

[33] Zhong Su, Hongjiang Zhang, Stan Li, Shaoping Ma, "Relevance Feedback in Content-Based Image Retrieval: Bayesian Framework, Feature Subspaces, and Progressive Learning", IEEE Transactions On Image Processing, Vol. 12, No. 8, August 2003, pp.924-937.

[34] S. Nene, S.Nayar, \& H. Murase. Columbia object image library (COIL-100). Technical report, CUCS-006-96, Feb 1996 http://www1.cs.columbia.edu/ CAVE/software/softlib/coil-100.php

[35] Ng, Raymond T.; Sedighian, Andishe, "Evaluating multidimensional indexing structures for images transformed by principal component analysis",Proc. SPIE Vol. 2670, p. 5061

[36] H.B.Kekre, Sudeep D. Thepade,AkshayMaloo, "Performance Comparison for Face Recognition using PCA, DCT \& Walsh Transform of Row Mean and Column Mean", ICGST International Journal on Graphics, Vision and Image Processing (GVIP), Volume 10, Issue 2, June 2010, pp.1927.

[37] C. Faloutsos, K. Lin, "FastMap: A Fast Algorithm for Indexing, DataMining and Visualization of Traditional and Multimedia Datasets", In Proc. of ACM SIG-MOD, pp.163174, 1995.

[38] Chandrasekaren S., Manjunath B., Wang Y., Winkler J., Zhang H., "An eigenspace update algorithm for image analysis", In Graphical Models and Image Processing, 1997.

[39] M. Kirby, L. Sirovich, Application of the Karhunen-Loeve Procedure for the Characterization of Human Faces, IEEE Transactions on Pattern Analysis and Machine Intelligence, Vol. 12, No. 1, January 1990, pp. 103-108

[40] H.B.Kekre, Sudeep D. Thepade, AkshayMaloo, "Performance Comparison of Image Retrieval Using Fractional Coefficients of Transformed Image Using DCT, Walsh, Haar and Kekre's Transform”, CSC International Journal of Image Processing (IJIP), Vol. 4 , Issue 2,
Computer Science Journals, CSC Press, www.cscjournals.org,

[41] H.B.Kekre, Sudeep D. Thepade, AkshayMaloo,"Eigenvectors of Covariance Matrix using Row Mean and Column Mean Sequences for Face Recognition", CSC International Journal International Journal of Biometric and Bioinformatics (IJBB), Vol. 4 , Issue 2, Computer Science Journals, www.cscjournals.org

[42] H.B.Kekre, Sudeep D. Thepade, AkshayMaloo, "Query by Image Content Using Colour Averaging Techniques", for International Journal of Engineering Science and Technology (IJEST), Volume 2, Issue 6, 2010. Available online at http://www.ijest.info

\section{AUTHOR PROFILE}

Dr. H. B. Kekre has received B.E. (Hons.) in Telecomm. Engineering. from Jabalpur University in 1958, M.Tech (Industrial Electronics) from IIT Bombay in 1960, M.S.Engg. (Electrical Engg.) from University of Ottawa in 1965 and Ph.D. (System Identification) from IIT Bombay in $1970 \mathrm{He}$ has worked as Faculty of Electrical Engg. and then HOD Computer Science and Engg. at IIT Bombay. For 13 years he was working as a professor and head in the Department of Computer Engg. At ThadomalShahani Engineering. College, Mumbai. Now he is Senior Professor at MPSTME, SVKM's NMIMS. He has guided 17 Ph.Ds, more than 100 M.E./M.Tech and several B.E./B.Tech projects. His areas of interest are Digital Signal processing, Image Processing and Computer Networking. He has more than 320 papers in National / International Conferences and Journals to his credit. He was Senior Member of IEEE. Presently He is Fellow of IETE and Life Member of ISTE Recently ten students working under his guidance have received best paper awards. Currently 10 research scholars are pursuing Ph.D. program under his guidance.

Sudeep D. Thepade has Received B.E.(Computer) degree from North Maharashtra University with Distinction in 2003. M.E. in Computer Engineering from University of Mumbai in 2008 with Distinction, currently pursuing Ph.D. from SVKM's NMIMS, Mumbai. He has about 07 years of experience in teaching and industry. He was Lecturer in Dept. of Information Technology at Thadomal Shahani Engineering College, Bandra (w), Mumbai for nearly 04 years. Currently working as Assistant Professor in Computer Engineering at Mukesh Patel School of Technology Management and Engineering, SVKM's NMIMS University, Vile Parle(w), Mumbai, INDIA. He is member of International Association of Engineers (IAENG) and International Association of Computer Science and Information Technology (IACSIT), Singapore. His areas of interest are Image Processing and Computer Networks. He has about 70 papers in National/International Conferences/Journals to his credit with a Best Paper Award at International Conference SSPCCIN-2008, Second Best Paper Award at ThinkQuest-2009 National Level paper presentation competition for faculty and Best Paper Award at Springer International Conference ICCCT-2010.

Akshay Maloo is currently pursuing B.Tech. (CS) from MPSTME, NMIMS University, Mumbai. His areas of interest are Artificial intelligence, Image Processing, Computer Networks and Databases. He has 7 papers in National/International Conferences/Journals to his credit. 